Research Article

\title{
Is There a Correlation between New Scoring Systems and Systemic Inflammation in Stable Bronchiectasis?
}

\author{
Hikmet Coban $^{1}$ and Adil Can Gungen ${ }^{2}$ \\ ${ }^{1}$ Department of Chest Disease, Sakarya Training and Research Hospital Sakarya, Adapazan, Turkey \\ ${ }^{2}$ Department of Chest Disease, Istinye University Istanbul, Istanbul, Turkey \\ Correspondence should be addressed to Hikmet Coban; hikmetcoban04@gmail.com
}

Received 12 July 2017; Accepted 19 October 2017; Published 15 November 2017

Academic Editor: Angelo G. Corsico

Copyright (c) 2017 Hikmet Coban and Adil Can Gungen. This is an open access article distributed under the Creative Commons Attribution License, which permits unrestricted use, distribution, and reproduction in any medium, provided the original work is properly cited.

\begin{abstract}
Aim. The present study aimed to investigate the relation between FACED and BSI scores, which were developed to measure the severity of bronchiectasis, and systemic inflammation in patients with stable bronchiectasis. Methods. FACED and BSI scores of 117 patients with stable bronchiectasis were calculated. The correlations between mean scores and CRP levels, leukocyte count, and neutrophil/lymphocyte ratio were investigated. Findings. Mean BSI and FACED scores were $7.2 \pm 5.2$ and $2.1 \pm 1.8$, respectively. The severity of bronchiectasis as determined based on BSI and FACED increased significantly with increasing levels of CRP in patients with stable bronchiectasis $(p=0.001$ and $p=0.027$, resp.). No significant changes were found in leukocyte count $(p=0.72$ and $p=0.09$, resp.) and N/L ratio $(p=0.45$ and $p=0.71$, resp.). BSI and FACED scores were significantly correlated with CRP but not with leukocyte count or N/L ratio. Conclusion. In patients with stable bronchiectasis who are evaluated based on FACED and BSI scores, CRP can be a useful biomarker as a direct indicator of the severity of systemic inflammation.
\end{abstract}

\section{Introduction}

Bronchiectasis is a chronic airway disease associated with symptoms such as cough, sputum production, and hemoptysis, and it may develop as a result of various etiologies [1]. A vicious cycle, based predominantly on airway infections, airway inflammation, and structural damage, plays a role in progression and pathogenesis of bronchiectasis [2]. It is disappointing that there are a limited number of effective treatment strategies and evidence-based management recommendations for assessment and follow-up of bronchiectasis. The severity of bronchiectasis should be determined to obtain better treatment outcomes. Assessment of bronchiectasis may be a challenge since there is actually no simple measurement method that has been sufficiently validated. Conventionally, previous reports measured the severity of disease based on FEV1 (forced expiratory volume in 1s) [3], while Reiff and Bhalla scores in HRCT were also used in some studies to measure disease severity $[4,5]$. However, FEV1 was not effective in terms of clinical decision-making, and HRCT (high-resolution computed tomography) scores had a poor correlation with lung functions [6]. Therefore, it became an obligation to create a new scoring system to assess the severity of bronchiectasis. FACED score (F: FEV1, A: age, C: colonization, E: number of affected lobes, and D: dyspnea) [7] and Bronchiectasis Severity Index (BSI) [8] were recently designed as two multidimensional severity grading scales to assess the prognosis of bronchiectasis. FACED score is a five-item grading system that predicts mortality in patients who had been monitored for 5 years. BSI is a seven-item scale that describes future risk of death, hospitalization, and exacerbations. Elevation of systemic inflammatory markers such as C-reactive protein (CRP) and total white blood cell count is known to be associated with the extent of the disease and poor lung functions [9]. The relation between CRP or total white blood cell count and the novel scoring systems used to assess bronchiectasis severity, namely, BSI and FACED, has not been investigated before.

Bronchiectasis is characterized by airway inflammation accompanied by continuous predominance of neutrophils. Proinflammatory cytokines such as IL-1, IL-6, and TNF-a increase, while anti-inflammatory cytokines such as IL-10 decrease in patients with bronchiectasis [10]. As 
a result of a series of complex interactions between various cells including the neutrophils and lymphocytes, inflammation may eventually cause permanent tissue damage [11]. The ratio of inflammatory cells in blood has a potential to reflect chronic inflammation and pathologic conditions, and the neutrophil/ lymphocyte ratio was recently shown to be increased during COPD exacerbations compared to stable disease conditions [12]. In the presence of various chronic conditions such as disorders of the cardiovascular and renal systems, it was shown that increased N/L ratio can be used as a marker of inflammation, and a higher N/L ratio was associated with disease severity, mortality, and hospitalization [13-15]. Since the lungs of patients with bronchiectasis are characterized by neutrophilic inflammation, N/L ratio can also be a useful marker to assess the clinical status of patients with bronchiectasis. The neutrophil/lymphocyte ratio is an inflammation marker that has not been previously investigated in patients with stable bronchiectasis. N/L ratio is an easily accessible marker that can be calculated from the total blood count, and it does not require any special equipment or specific method of measurement. N/L ratio can become an easy-to-use method and a useful marker in patients with bronchiectasis if it proves to be useful as a biological marker in this patient group.

The present study aimed to investigate the relation of CRP, total white blood cell count, and neutrophil/ lymphocyte ratio with BSI and FACED scores.

\section{Methods}

Patients who had been followed up by Pulmonology Clinics of Sakarya Training and Research Hospital and had a definitive diagnosis of bronchiectasis based on HRCT and BTS guidelines were included to the study [16]. The diagnosis of bronchiectasis with HRCT is based on radiology reports. BTS guideline mentions investigation and management of patients with bronchiectasis. BTS guideline does not include bronchiectasis caused by cystic fibrosis [16]. Patients with immunodeficiency, allergic pulmonary aspergillosis, bronchiectasis secondary to primary ciliary dyskinesia, heart failure, malignancy or chronic renal failure; patients who had used antibiotics or steroids within the last one month; patients who described an acute exacerbation; and pregnant patients were excluded from the study. A total number of 117 patients who met the above-described criteria were included to the study. These patients were followed between 2014 and 2016. The patients who had a history of steroid and antibiotic use in the last month were evaluated in the stabilization period of bronchiectasis. All patients performed spirometry, and peripheral venous blood samples were obtained from all patients on the day of spirometry. Total blood count and CRP levels were analyzed. In patients who were following up for two years, at least two measurements of CRP, WBC, and N/L were taken in the stabilization period, and the averages of these measurements were calculated. For the measurement of serum CRP levels, $3 \mathrm{cc}$ of venous blood samples were collected in biochemistry test tubes. Blood samples were transferred to the analyzing laboratory at most 2 hours after sampling, and the analyses were performed on the same day. CRP levels were quantitatively analyzed by the nephelometric method using the BN 200 device. The reference range in healthy humans for the preferred method of analyses was $0.00-5.00 \mathrm{mg} / \mathrm{L}$.

2.1. FACED and BSI Scoring and Grading. FACED score consists of 5 variables: FEV 1\%, age, Pseudomonas aeruginosa colonization, radiological extent of the disease, and dyspnea as assessed by the Medical Research Council (MRC) scale. The total score is calculated by adding up the scores for each variable and varies between 0 and 7 points. Based on the total score, bronchiectasis is evaluated in three groups as mild (0-2), moderate [3, 4], and severe [5-7] bronchiectasis.

BSI score consists of 9 variables: age, body mass index, FEV1\%, hospitalizations within the last two years, number of exacerbations within the last one year, dyspnea as assessed by MRC scale, Pseudomonas aeruginosa colonization, colonization by other microorganisms, radiological extent of the disease, and/or presence of cystic bronchiectasis. Total score varies between 0 and 26 points. In BSI, scores of $0-4$ points indicate mild, 5-8 indicate moderate, and 9 or above indicate severe bronchiectasis.

2.2. Statistical Methods. SPSS for Windows 18.0 (Chicago, IL) software package was used for statistical analyses. Continuous variables were presented as mean values and standard deviations. Differences between two groups were tested by $t$-test for normally distributed variables and by Mann-Whitney test for nonnormally distributed variables. For analysis of more than two groups, ANOVA test was used for normally distributed variables and Kruskal-Wallis test was used for nonnormally distributed variables.

Correlations were evaluated by means of Spearman's rank correlation test. A $p$ value less than 0.05 was considered as significant.

\section{Results}

Table 1 shows the characteristics of 117 patients with stable bronchiectasis who were included in this study. Mean FACED and BSI scores and numbers of patients with mild, moderate, and severe bronchiectasis based on both scoring systems are shown in Tables 2 and 3.

When the patients were classified as those with mild, moderate, and severe bronchiectasis according to BSI and FACED scores, significant relations were found between FVC $\%$, FEV1\%, FEV1/FVC, CRP, BMI, number of exacerbations, and number of hospitalizations according to both scoring systems. WBC and N/L ratio were not significantly related to disease severity based on neither of the scoring systems (Tables 2 and 3). A significant positive correlation was found between FACED and BSI scores and CRP levels (Figure 1), and a significant negative correlation was noted between pulmonary function test parameters and BMI index. No significant correlation was demonstrated between FACED and BSI scores and N/L ratio or leukocyte count (Table 4). Comorbid conditions which may have an effect on systemic inflammation and the CRP levels are shown in Table 5. There was no significant difference between the groups. 
TABLE 1: Characteristics of the study participants.

\begin{tabular}{lc}
\hline Characteristics & Patients $(n=117)$ \\
\hline Male/female (N\%) & $65 / 52(55.6 / 44.4)$ \\
Mean age (years) & $53 \pm 15.1$ \\
BMI (kg/m $\left.{ }^{2}\right)$ & $27.3 \pm 5.6$ \\
FVC (\%) & $64.2 \pm 21.2$ \\
FEV1 (\%) & $55.5 \pm 21.2$ \\
FEV1/FVC (\%) & $70.1 \pm 13.7$ \\
Pack-years smoked & $9.7 \pm 14.8$ \\
Ex-smokers, $N(\%)$ & $30(25.6)$ \\
Never smokers, $N(\%)$ & $68(58.1)$ \\
Current smokers, $N(\%)$ & $19(15.3)$ \\
CRP (mg/L) & $10.4 \pm 8.0$ \\
N/L ratio & $2.2 \pm 1.5$ \\
WBC (/mm $\left.{ }^{3}\right)$ & $8449 \pm 2449$ \\
Pseudomonas aeruginosa, $N(\%)$ & $11(9.4)$ \\
Other microorganisms, $N(\%)$ & $11(9.4)$ \\
Number of affected lobes & $2.6 \pm 1.0$ \\
Exacerbations in previous year & $2.0 \pm 2.3$ \\
Number of hospitalizations in the last & $0.3 \pm 0.6$ \\
two years &
\end{tabular}

BMI, body mass index; FVC, forced vital capacity; FEV1, forced expiratory volume in 1 second; FEV1/FVC, forced expiratory volume in 1 second/forced vital capacity; N/L ratio, neutrophil/lymphocyte ratio.

TABLE 2: The relation between bronchiectasis severity based on BSI score and the other study variables.

\begin{tabular}{|c|c|c|c|c|}
\hline \multicolumn{5}{|c|}{ BSI score $=7.2 \pm 5.2$} \\
\hline & $\begin{array}{c}\text { Mild } \\
(n=46)\end{array}$ & $\begin{array}{c}\text { Moderate } \\
(n=25)\end{array}$ & $\begin{array}{c}\text { Severe } \\
(n=46)\end{array}$ & $p$ \\
\hline Age (years) & 47.1 & 56.1 & 57.2 & 0.003 \\
\hline FVC (\%) & 82.6 & 55.0 & 52.1 & $<0.0001$ \\
\hline FEV1 (\%) & 74.7 & 46.7 & 42.5 & $<0.0001$ \\
\hline FEV1/FVC (\%) & 74.4 & 71.0 & 65.8 & 0.020 \\
\hline CRP (mg/L) & 6.8 & 12.9 & 12.5 & 0.001 \\
\hline $\mathrm{WBC}\left(/ \mathrm{mm}^{3}\right)$ & 8224 & 8631 & 8574 & 0.727 \\
\hline $\mathrm{N} / \mathrm{L}$ ratio & 2.36 & 2.40 & 2.08 & 0.456 \\
\hline Smoking (pack-years) & 9.2 & 12.0 & 9.0 & 0.718 \\
\hline BMI $\left(\mathrm{kg} / \mathrm{m}^{2}\right)$ & 29.5 & 25.4 & 26.2 & 0.008 \\
\hline $\begin{array}{l}\text { Exacerbations in } \\
\text { previous year }\end{array}$ & 0.28 & 2.44 & 3.65 & $<0.0001$ \\
\hline $\begin{array}{l}\text { Number of } \\
\text { hospitalizations } \\
\text { in the last two years }\end{array}$ & 0.00 & 0.40 & 0.95 & $<0.0001$ \\
\hline
\end{tabular}

FVC, forced vital capacity; FEV1, forced expiratory volume in 1 second; FEV1/FVC, forced expiratory volume in 1 second/forced vital capacity; N/L ratio, neutrophil/lymphocyte ratio; BMI, body mass index.

\section{Discussion}

In the present study, the severity of bronchiectasis was assessed based on the recently developed FACED and BSI scoring systems, while systemic inflammation was evaluated by CRP levels, leukocyte count, and N/L ratio. Analyses of
TABLE 3: The relation between bronchiectasis severity based on FACED score and the other study variables.

\begin{tabular}{|c|c|c|c|c|}
\hline \multicolumn{5}{|c|}{ FACED score $=2.1 \pm 1.8$} \\
\hline & $\begin{array}{l}\text { Mild } \\
(n=71)\end{array}$ & $\begin{array}{c}\text { Moderate } \\
(n=34)\end{array}$ & $\begin{array}{c}\text { Severe } \\
(n=12)\end{array}$ & $p$ \\
\hline Age (years) & 48.3 & 56.6 & 70.1 & $<0.0001$ \\
\hline $\mathrm{FVC}(\%)$ & 76.1 & 51.4 & 40.0 & $<0.0001$ \\
\hline FEV1 $(\%)$ & 68.2 & 39.9 & 35.0 & $<0.0001$ \\
\hline FEV1/FVC (\%) & 74.0 & 61.0 & 73 & $<0.0001$ \\
\hline $\mathrm{CRP}(\mathrm{mg} / \mathrm{L})$ & 8.8 & 12.4 & 13.8 & 0.027 \\
\hline $\mathrm{WBC}\left(/ \mathrm{mm}^{3}\right)$ & 8190 & 9197 & 7858 & 0.096 \\
\hline $\mathrm{N} / \mathrm{L}$ ratio & 2.26 & 2.35 & 2.01 & 0.715 \\
\hline Smoking (pack-years) & 8.4 & 12.0 & 12.0 & 0.488 \\
\hline BMI $\left(\mathrm{kg} / \mathrm{m}^{2}\right)$ & 28.6 & 24.5 & 27.4 & 0.007 \\
\hline $\begin{array}{l}\text { Exacerbations in } \\
\text { previous year }\end{array}$ & 1.3 & 3.2 & 3.0 & $<0.0001$ \\
\hline $\begin{array}{l}\text { Number of } \\
\text { hospitalizations } \\
\text { in the last two years }\end{array}$ & 0.15 & 0.64 & 1.0 & $<0.0001$ \\
\hline
\end{tabular}

FACED (F: forced expiratory volume in 1 second, A: age, C: colonization, E: number of affected lobes, D: dyspnea); FVC, forced vital capacity; FEV1, forced expiratory volume in 1 second; FEV1/FVC, forced expiratory volume in 1 second/forced vital capacity; N/L ratio, neutrophil/lymphocyte ratio; BMI, body mass index.

the correlations between FACED and BSI scores and markers of systemic inflammation in patients with stable bronchiectasis demonstrated a significant relation only with CRP levels, whereas no significant correlation was found with leukocyte count or N/L ratio (Table 4).

A study assessing the severity of bronchiectasis based on HRCT score reported a significant correlation between HRCT score and markers of systemic inflammation such as WBC and CRP [9]. In a study involving patients with diseases that result in respiratory failure, patients with bronchiectasis were shown to have higher CRP levels compared to the other patient groups, and CRP levels were associated with mortality in that study [17]. Patients with bronchiectasis and elevated CRP levels showed rapid FEV1 declines in another study [18]. All these findings suggest that, as long as bronchiectasis is accompanied by systemic inflammation, systemic inflammation should presumably be associated with disease severity. Previous studies assessed the severity of bronchiectasis based on FEV1 or HRCT scores. No previous study demonstrated a correlation between CRP and the recently developed BSI or FACED scoring systems. In the present study, CRP had a significant positive correlation both with BSI $(r=0.30, p=0.001)$ and FACED $(r=0.30, p=0.001)$ scores. Contrary to our findings, a previous study comparing patients with bronchiectasis to a control group did not identify any finding indicative of systemic inflammation in the bronchiectasis group, and the authors highlighted that increased WBC count and CRP levels in patients with colonization might suggest presence of an inflammatory response [19].

It has been emphasized that increased bronchial inflammation may be present even during periods of clinical 

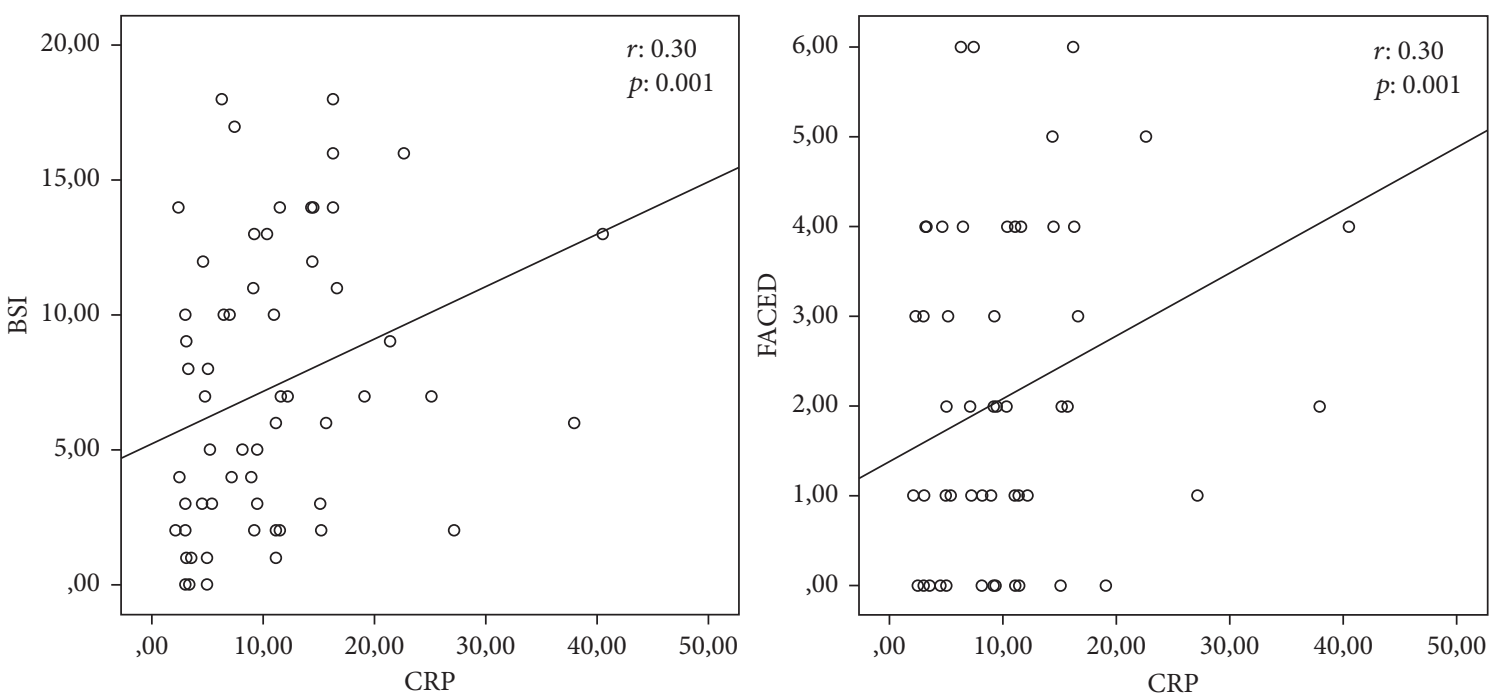

FIgURE 1: Correlation FACED and BSI scores with CRP levels.

TABLE 4: Correlation coefficients and $p$ values for FACED and BSI scores and study variables.

\begin{tabular}{lcccc}
\hline & \multicolumn{2}{c}{ FACED score } & \multicolumn{2}{c}{ BSI score } \\
& $r$ & $p$ & $r$ & $p$ \\
\hline FVC $(\%)$ & -0.70 & $<0.0001$ & -0.62 & $<0.0001$ \\
FEV1 $(\%)$ & -0.73 & $<0.0001$ & -0.67 & $<0.0001$ \\
WBC $\left(/ \mathrm{mm}^{3}\right)$ & 0.16 & 0.075 & 0.01 & 0.850 \\
CRP & 0.30 & 0.001 & 0.30 & 0.001 \\
N/L ratio & -0.02 & 0.822 & -0.11 & 0.238 \\
BMI $\left(\mathrm{kg} / \mathrm{m}^{2}\right)$ & -0.32 & 0.002 & -0.21 & 0.047 \\
\hline
\end{tabular}

FACED (F: forced expiratory volume in 1 second, A: age, C: colonization, E: number of affected lobes, D: dyspnea); BSI, Bronchiectasis Severity Index; FVC, forced vital capacity; FEV1, forced expiratory volume in 1 second; N/L ratio, neutrophil/lymphocyte ratio.

TABle 5: Comorbid conditions, which may have an effect on systemic inflammation and the CRP levels.

\begin{tabular}{lcc}
\hline & CRP $(\mathrm{mg} / \mathrm{L})$ & $p$ \\
\hline Total $(n=117)$ & $10.4 \pm 8.0$ & \\
COPD $(n=23)$ & $12.8 \pm 6.9$ & \\
Asthma $(n=38)$ & $10.0 \pm 7.6$ & 0.30 \\
Hypertension $(n=7)$ & $9.8 \pm 7.1$ & \\
DM $(n=6)$ & $4.9 \pm 1.4$ & \\
None $(n=43)$ & $10.2 \pm 9.4$ & \\
\hline
\end{tabular}

COPD, chronic obstructive pulmonary disease; DM, diabetes mellitus; CRP, C-reactive protein.

stability. During exacerbations, neutrophils migrate to the airways and proteolytic activity increases. Proteolytic agents destroy the lung matrix and contribute to the development of bronchiectasis [20]. In patients with stable bronchiectasis, presence of neutrophilic inflammation in sputum was suggested to be a good biomarker of disease severity. In that study, disease severity was assessed based on lung functions and BSI [21]. Considering there is an inflammation in the lungs with neutrophil predominance, demonstration of this condition in systemic circulation can be a useful parameter to grade the disease severity. N/L ratio was assessed as a marker of systemic inflammation in the presence of chronic diseases involving cardiovascular and renal systems [13-15]. Since bronchiectasis is also a chronic disease, one might expect that the increased neutrophilic inflammation in the lungs might be associated with an elevated N/L ratio in systemic circulation with increasing disease severity. No previous study in the literature investigated N/L ratio in patients with stable bronchiectasis. In the present study, a significant correlation was not found between N/L ratio and FACED or BSI scores in patients with stable bronchiectasis.

In a study performed on patients with last-stage respiratory disorders, including 33 patients with bronchiectasis, a clear relation was demonstrated between decreased BMI and increased mortality [18]. Similarly, a Turkish study suggested that higher BMI values could be beneficial on expected survival time in patients with bronchiectasis [22]. BMI was associated with the findings that reflect disease severity. Patients with low BMI values were shown to more frequently experience acute exacerbations, have poor lung functions, have increased systemic inflammation, and more commonly have chronic P. aeruginosa colonization. BMI was emphasized to be one of the factors with the most significant effects on the risk of hospitalization and death [18, 22]. A positive correlation was demonstrated between high BMI and survival [23]. A recent study reported a relation between radiological progression and low BMI [24]. In the present study, significant relations were found between BMI and both BSI and FACED severity classifications reflecting the severity of bronchiectasis. Both scoring systems had significant negative correlations with BMI, while the correlation with FACED $(r=-0.32, p=0.002)$ score was more evident than the correlation with BSI $(r=-0.21, p=0.047)$ score.

A recently published study reported that low FVC values represented a risk factor for bacterial colonization [25]. Both BSI and FACED scorings take into account the FEV1 values. 
Since both scoring systems had significant negative correlations with FVC in the present study (FACED $(r=-0.70$, $p<0.0001)$, BSI $(r=-0.62, p<0.0001)$ ), it should be considered that the risk of bacterial colonization may increase with numeric increases in both scores, in other words, with increasing disease severity.

Patient distribution was different when the disease severity was classified as mild, moderate, or severe based on the two scoring systems in the present study. On the contrary, Minov et al. [26] reported similar patient distribution rates based on both scoring systems (Tables 2 and 3 ). To explain this, in a recently published study, Guan et al. emphasized that the difference in FEV1 distribution and the scoring for previous hospitalizations resulted in overall higher BSI scores; therefore, some patients were given a higher severity grade. The authors underlined the need for additional studies comparing effectiveness of grading systems in bronchiectasis [27].

This study has some limitations. Since the study was performed as a single-center study with a small sample size, study results can only be generalized to a limited extent. Moreover, the groups were not homogenous in terms of severity grading based on the two scoring systems.

\section{Conclusion}

In patients with bronchiectasis, CRP can be a useful biomarker that directly reflects the level of systemic inflammation. WBC and N/L ratio failed to capture systemic inflammation in patients with stable bronchiectasis whose severity grading was done based on FACED and BSI scoring systems. Additional studies are required to elucidate the clinical significance of the role of CRP in the assessment of treatment response and progression of bronchiectasis after anti-inflammatory therapy.

\section{Conflicts of Interest}

The authors declare that there are no conflicts of interest regarding the publication of this paper.

\section{References}

[1] W. J. Guan, Y. H. Gao, G. Xu et al., "Aetiology of bronchiectasis in Guangzhou, southern China," Respirology, vol. 20, no. 5, pp. 739-748, 2015.

[2] J. D. Chalmers, M. P. Smith, B. J. McHugh, C. Doherty, J. R. Govan, and A. T. Hill, "Short and long term antibiotic treatment reduces airway and systemic inflammation in non-cystic fibrosis bronchiectasis," American Journal of Respiratory and Critical Care Medicine, vol. 186, no. 7, pp. 657-665, 2012.

[3] A. Torrego, R. A. Haque, L. T. Nguyen et al., "Capsaicin cough sensitivity in bronchiectasis," Thorax, vol. 61, no. 8, pp. 706-709, 2006.

[4] D. B. Reiff, A. U. Wells, D. H. Carr, P. J. Cole, and D. M. Hansell, "CT findings in bronchiectasis: limited value in distinguishing between idiopathic and specific types," American Journal of Roentgenology, vol. 165, no. 2, pp. 261-267, 1995.
[5] M. Bhalla, N. Turcios, V. Aponte et al., "Cystic fibrosis: scoring system with thin- section CT," Radiology, vol. 179, no. 3, pp. 783-788, 1991.

[6] M. Restrepo and J. S. Alborn, "Bronchiectasis severity: time to score," American Journal of Respiratory and Critical Care Medicine, vol. 189, no. 5, pp. 508-509, 2014.

[7] M. A. Martinez-Garcia, J. de Gracia, M. Vendrell Relat et al., "Multidimensional approach to non-cystic fibrosis bronchiectasis: the FACED score," European Respiratory Journal, vol. 43, no. 5, pp. 1357-1367, 2014.

[8] J. D. Chalmers, P. Goeminne, S. Aliberti et al., "The bronchiectasis severity index: an international derivation and validation study," American Journal of Respiratory and Critical Care Medicine, vol. 189, no. 5, pp. 576-585, 2014.

[9] C. B. Wilson, P. W. Jones, C. J. O'Leary et al., "Systemic markers of inflammation in stable bronchiectasis," European Respiratory Journal, vol. 12, no. 4, pp. 820-824, 1998.

[10] S. Fuschillo, A. de Felice, and G. Balzano, "Mucosal inflammation in idiopathic bronchiectasis: cellular and molecular mechanisms," European Respiratory Journal, vol. 31, no. 2, pp. 396-406, 2008.

[11] C. Nathan, "Points of control in inflammation," Nature, vol. 420, no. 6917, pp. 846-852, 2002.

[12] E. Günay, S. Sarinc Ulali, O. Akar et al., "Neutrophilto lymphocyte ratio in chronic obstructive pulmonary disease: a retrospective study," Inflammation, vol. 37, no. 2, pp. 374-380, 2014.

[13] R. Zahorec, "Ratio of neutrophil to lymphocyte counts: rapid and simple parameter of systemic inflammation and stress in critically ill," Bratislavské Lekárske Listy, vol. 102, no. 1, pp. 5-14, 2001.

[14.] B. K. Duffy, H. S. Gurm, V. Rajagopal, R. Gupta, and S. G. Ellis, "Usefulness of an elevated neutrophil to lymphocyte ratio in predicting long-term mortality after percutaneous coronary intervention," American Journal of Cardiology, vol. 97, no. 7, pp. 993-996, 2006.

[15] Y. Arbel, A. Finkelstein, A. Halkin et al., "Neutrophil/ lymphocyte ratio is related to the severity of coronary artery disease and clinical outcome in patients undergoing angiography," Atherosclerosis, vol. 225, no. 2, pp. 456-460, 2012.

[16] M. C. Pasteur, D. Bilton, A. T. Hill, and British Thoracic Society Bronchiectasis non-CF Guideline Group, "British Thoracic Society guideline for non-CF bronchiectasis," Thorax, vol. 65, no. 1, pp. i1-i58, 2010.

[17] M. A. Martínez-García, J. J. Soler-Cataluña, M. PerpiñáTordera, P. Román-Sánchez, and J. Soriano, "Factors associated with lung function decline in adult patients with stable non-cystic fibrosis bronchiectasis," Chest, vol. 132, no. 5, pp. 1565-1572, 2007.

[18] N. J. M. Cano, C. Pichard, H. Roth et al., "C-reactive protein and body mass index predict outcome in end-stage respiratory failure," Chest, vol. 126, no. 2, pp. 540-546, 2004.

[19] B. Ergan Arsava and L. Cöplü, "Does airway colonization cause systemic inflammation in bronchiectasis?," Tuberkuloz ve Toraks, vol. 59, no. 4, pp. 340-347, 2011.

[20] M. Gaga, A. M. Bentley, M. Humbert et al., "Increases in CD4 + T lymphocytes, macrophages, neutrophils and interleukin 8 positive cells in the airways of patients with bronchiectasis," Thorax, vol. 53, no. 8, pp. 685-691, 1998.

[21] F. L. Dente, M. Bilotta, M. L. Bartoli et al., "Neutrophilic bronchial inflammation correlates with clinical and functional findings in patients with noncystic fibrosis bronchiectasis," Mediators of Inflammation, vol. 2015, Article ID 642503, 6 pages, 2015. 
[22] Q. Qi, T. Li, J. C. Li, and Y. Li, "Association of body mass index with disease severity and prognosis in patients with non-cystic fibrosis bronchiectasis," Brazilian Journal of Medical and Biological Research, vol. 48, no. 8, pp. 715-724, 2015.

[23] Z. P. Onen, B. E. Gulbay, E. Sen et al., "Analysis of the factors related to mortality in patients with bronchiectasis," Respiratory Medicine, vol. 101, no. 7, pp. 1390-1397, 2007.

[24] J. Park, S. Kim, Y. J. Lee et al., "Factors associated with radiologic progression of non-cystic fibrosis bronchiectasis during long-term follow-up," Respirology, vol. 21, no. 6, pp. 1049-1054, 2016.

[25] S. Borekci, A. N. Halis, G. Aygun, and B. Musellim, "Bacterial colonization and associated factors in patients with bronchiectasis," Annals of Thoracic Medicine, vol. 11, no. 1, pp. 55-59, 2016.

[26] J. Minov, J. Karadzinska-Bislimovska, K. Vasilevska, S. Stoleski, and D. Mijakoski, "Assessment of the non-cystic fibrosis bronchiectasis severity: the FACED score vs the bronchiectasis severity index," Open Respiratory Medicine Journal, vol. 9, no. 1, pp. 46-51, 2015.

[27] W. J. Guan, R. C. Chen, and N. S. Zhong, "The bronchiectasis severity index and FACED score for bronchiectasis," European Respiratory Journal, vol. 47, no. 2, pp. 382-384, 2016. 


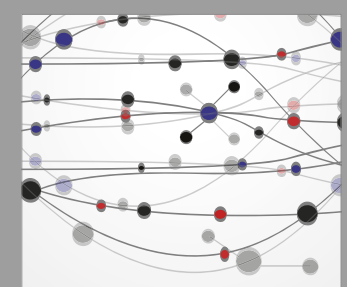

The Scientific World Journal
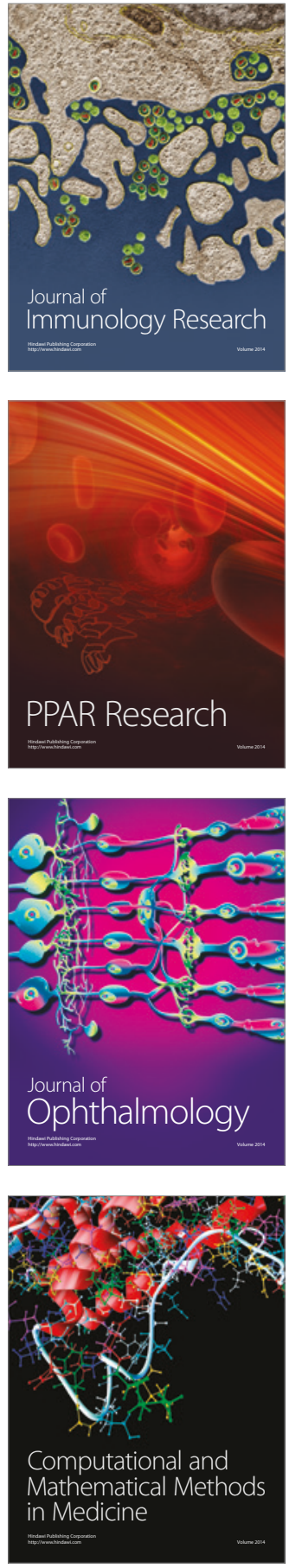

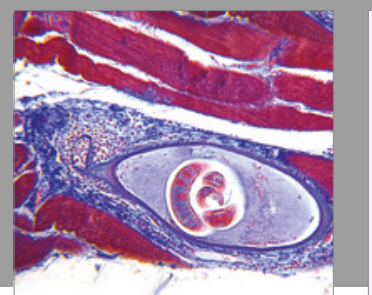

Gastroenterology Research and Practice
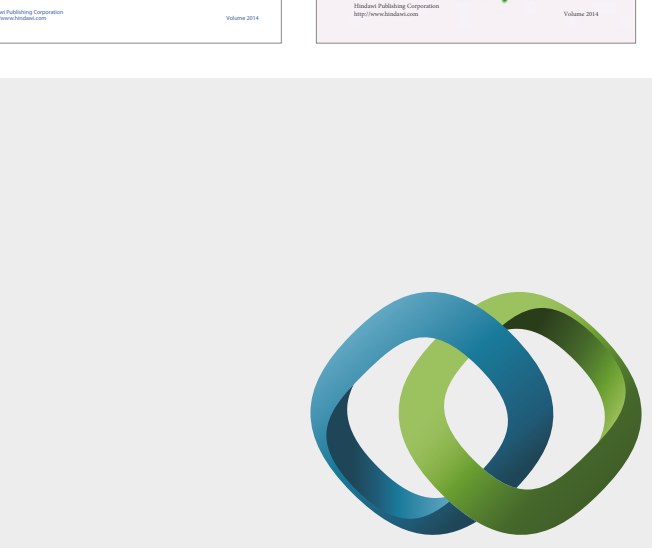

\section{Hindawi}

Submit your manuscripts at

https://www.hindawi.com
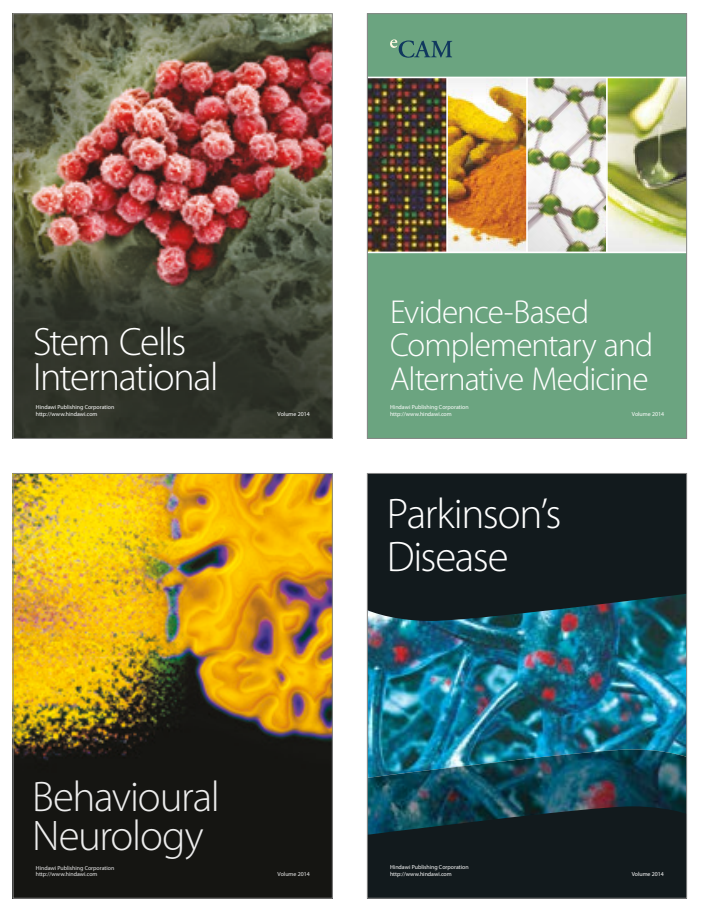
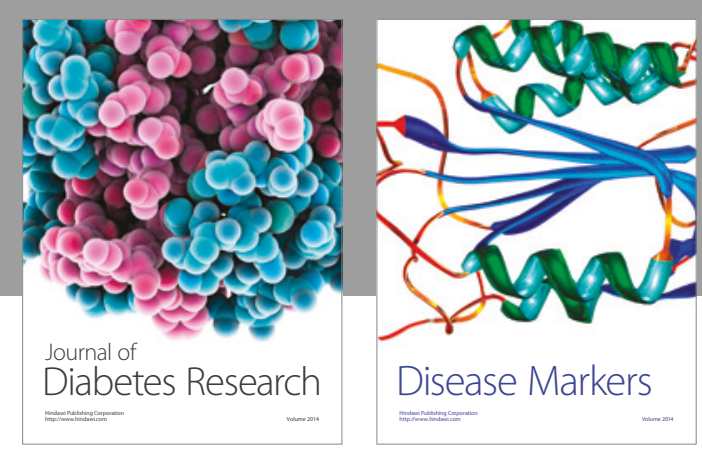

Disease Markers
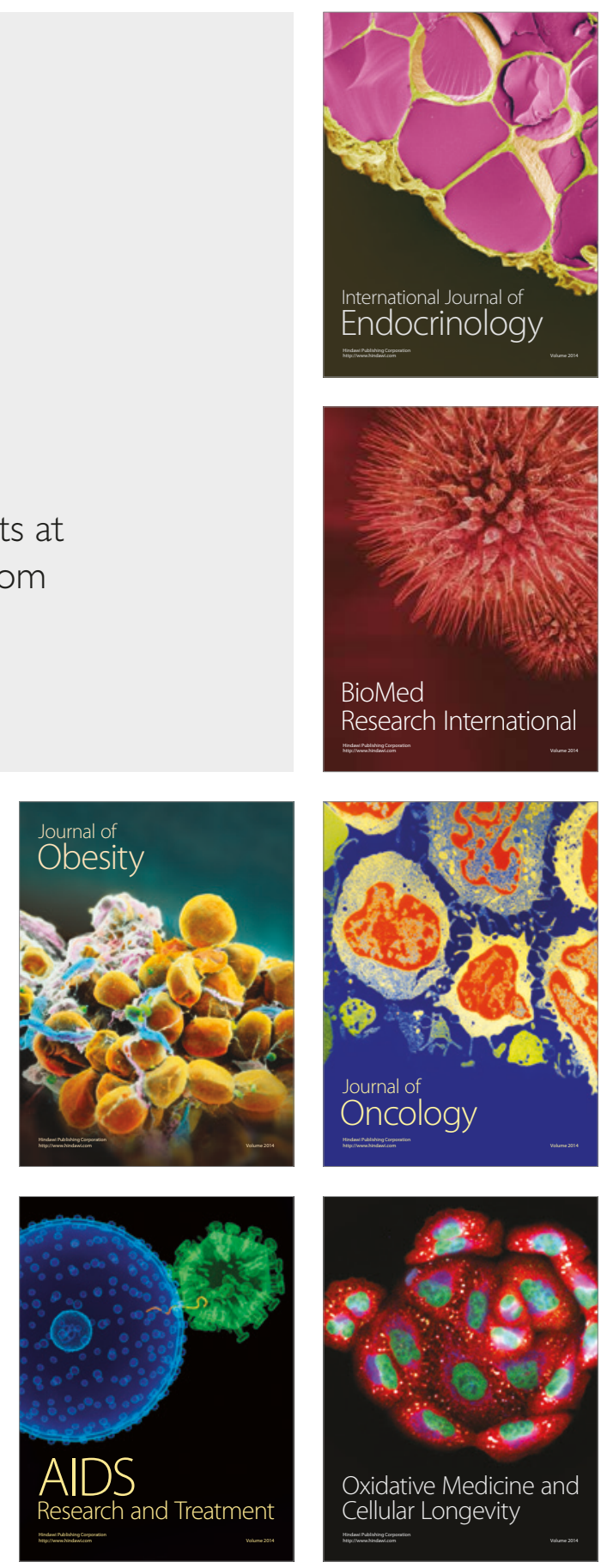